\title{
Search for Cosmic Strings in the GOODS Survey
}

\author{
J. L. Christiansen* E. Albin, and K. A. James \\ Department of Physics, California Polytechnic State University, San Luis Obispo, California 93407, USA \\ J. Goldman \\ Department of Physics, Faculty of Science, National University of Singapore, Singapore, 117542 \\ D. Maruyama \\ Department of Physics, University of California, Berkeley, California 94720, USA \\ G. F. Smoot \\ Lawrence Berkeley National Laboratory, Space Sciences Laboratory \\ and Department of Physics, University of California, Berkeley, California 94720, USA
}

(Dated: October 25, 2018)

\begin{abstract}
We search Hubble Space Telescope Treasury Program images collected as part of the Great Observatories Origins Deep Survey for pairs of galaxies consistent with the gravitational lensing signature of a cosmic string. Our technique includes estimates of the efficiency for finding the lensed galaxy pair. In the north (south) survey field we find no evidence out to a redshift of greater than $0.5(0.3)$ for cosmic strings to a mass per unit length limit of $G \mu / c^{2}<3.0 \times 10^{-7}$ at $95 \%$ confidence limits (C.L.). In the combined $314.9 \mathrm{arcmin}^{2}$ of the north and south survey fields this corresponds to a global limit on $\Omega_{\text {strings }}<0.02$. Our limit on $G \mu / c^{2}$ is more than an order of magnitude lower than searches for individual strings in cosmic microwave background (CMB) data. Our limit is higher than other CMB and gravitational wave searches, however, we note that it is less model dependent than these other searches.
\end{abstract}

PACS numbers: $98.80 . \mathrm{Cq}$

\section{INTRODUCTION:}

Cosmic strings can be formed during symmetrybreaking phase transitions in the early universe [1, 2]. Their well defined equations of motion and interaction potentials give us reason to believe that they have evolved into a modernday string network, observable through a variety of astrophysical phenomena [3]. Proposed as a natural consequence of the cooling universe, they were originally believed to be an unavoidable byproduct of symmetry breaking at the grand unified theory (GUT) scale and it was thought that measurement of their mass per unit length would tell us the temperature of phase transitions. More recently, cosmic (super) strings have been proposed as a byproduct of supersymmetric F- or D-term inflation, occurring after the GUT scale transition and resulting in a stochastic network with interaction probabilities less than 1 due to extra dimensions that allow strings to pass without touching [4]. In either case, the dimensionless scale of observational interest is from $10^{-6} \gtrsim G \mu / c^{2} \gtrsim 10^{-11}$.

Although there has been considerable interest within the theory community, only a few observations bear on the subject: (1) cosmic microwave background (CMB), (2) gravitational waves, (3) gravitational lensing. The CMB power spectrum shows that cosmic strings are not

*Electronic address: jlchrist@calpoly.edu the dominant factor in large-scale structure formation, contributing less than $10 \%$ of the observed structure [5, 6, 7, 8]. Searches for individual strings in the CMB set a limit $G \mu / c^{2} \lesssim 3.7 \times 10^{-6}[9,10]$. Bursts of gravitational waves are predicted from cusps in cosmic strings as they acquire a large Lorentz boost due to the string tension. A population of cusps and loops is expected to produce a stochastic background of gravitational waves that can be detected via pulsar timing and also by direct measurement with LIGO 11, 12]. The lack of a gravitational wave signal sets a limit on cosmic strings masses, $G \mu / c^{2} \lesssim 1.5 \times 10^{-8}$. This limit depends on properties of the string network such as the physical model, number, and strength of interactions. Gravitational lensing by a cosmic string of background galaxies has also been considered. A candidate pair of morphologically similar galaxies, CSL-1, was discovered in March of 2002 [13, 14] but follow-up Hubble images proved it to be a binary system [15]. A systematic search of an optical survey field as we report here has not previously been published.

Our aim in this paper is to use the wide-and-deepfield survey carried out by the Great Observatories Origins Deep Survey (GOODS) team with the Hubble Space Telescope (HST) Advanced Camera for Surveys (ACS) to search for the existence of cosmic strings using their lensing signature. We have developed the observational technique beyond what has been previously attempted [13, 16] by including the efficiencies of finding the lensed galaxy in our analysis. We note that the string masses excluded with this technique are lower than those ruled 
out by the CMB search for individual strings. Still, the CMB full-sky observations at very large redshifts search a larger volume of the universe. Searching for less massive strings in high resolution wide-field surveys is important, however, because in many models the density of strings increases logarithmically with decreasing mass. Recently reported sensitivities to cosmic strings suggest that modern optical surveys are competitive with other methods [17]. At the present time, limiting the mass scale and density of cosmic strings is important for model development. Ultimately, the discovery of cosmic strings would be an important key to the physics of the early universe.

In Sec. II we give an overview of the lensing technique. Section III follows with a description of our data selection. Section IV then describes the simulation of galaxy lensing by cosmic strings that is used in Sec. V to determine our detection efficiencies. The efficiencies are used in Sec. VI to determine limits on individual cosmic strings as a function of mass and redshift as well as the limit on the density of cosmic strings in the universe. We summarize our results in Sec. VII.

\section{OVERVIEW OF LENSING TECHNIQUE:}

The geometry of space is altered by the large mass per unit length of a cosmic string. A long straight cosmic string will cause an angular defect or deficit according to

$$
d s^{2}=d z^{2}+d r^{2}+\left(1-4 \frac{G \mu}{c^{2}}\right)^{2} r^{2} d \theta^{2}
$$

where the coordinate $z$ is along the string, and $r$ and $\theta$ are the polar coordinates of a plane perpendicular to the string [18]. The deficit angle is given by the dimensionless parameter, $\delta=8 \pi \frac{G \mu}{c^{2}}$ which results in the lensing effect on background galaxies, making identical pairs appear on both sides of the string. The opening angle between the two observed images is related to the deficit angle by

$$
\Delta \theta=\delta \sin (\beta) \frac{D_{l s}}{D_{o s}}
$$

where $D_{l s}$ is the distance between the lensing string (l), and the background source (s), $D_{o s}$ is the distance between the observer (o) and the background source, and $\beta$ is the tilt of the string toward the observer [19].

Our strategy is to search the GOODS wide-field survey for all pairs of galaxies that are morphologically similar - the hallmark of a cosmic string - with opening angles less than $15^{\prime \prime}$. Figure 1 shows simulated pairs of galaxies produced by massive cosmic strings at redshifts of 0.5 and 1.0 in a small part of the survey. In contrast, Fig. 2 shows the random pairs of morphologically similar galaxy pairs that comprise the background to the cosmic string search.

The difference between the signal and background is characterized statistically by the number of morphologically similar galaxy pairs as a function of the opening

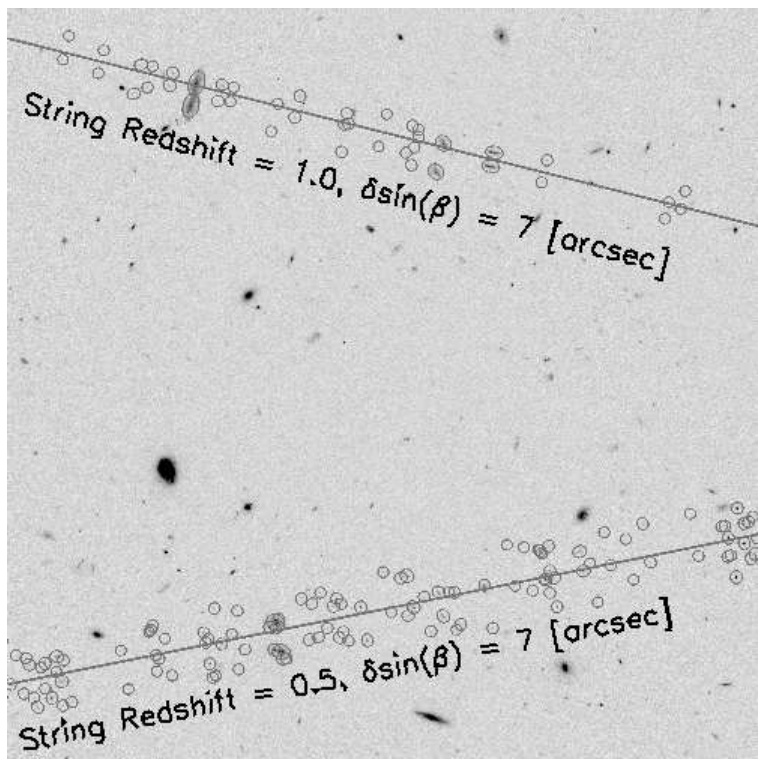

FIG. 1: Simulated cosmic strings at redshift 0.5 and 1.0 in a small part of the GOODS north field. Pairs of morphologically similar galaxies are expected to fall on opposite sides of the string.

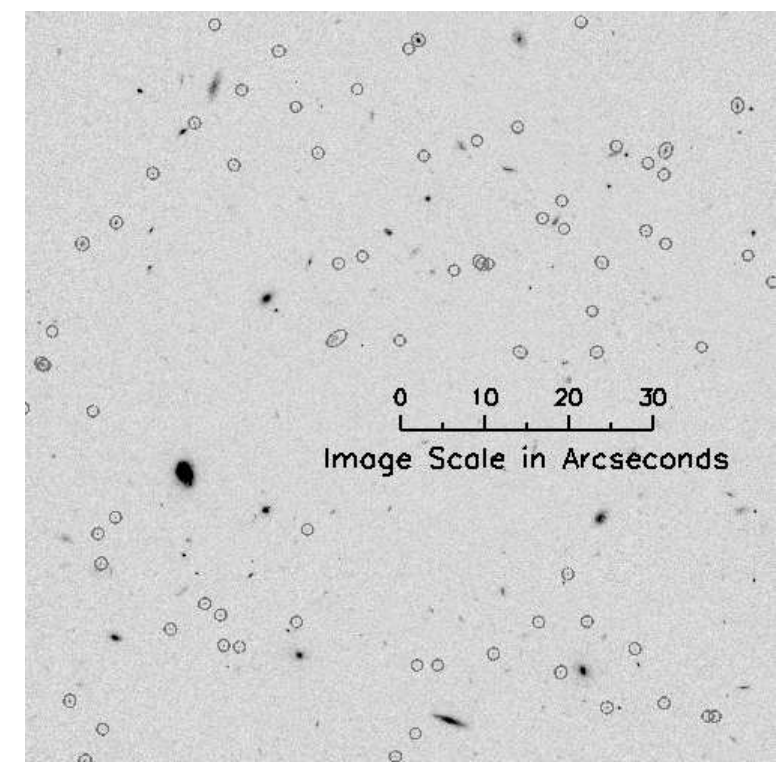

FIG. 2: Pairs of morphologically similar galaxies found in a region of the GOODS survey. These pairs form the background to the signal pairs from a cosmic string.

angle. Pairs associated with a string are expected to pile up at angles, $\Delta \theta$, less than about $6^{\prime \prime}$. The distribution depends on mass, redshift, and tilt of the string as well as specifics of the survey which are further discussed in Sec. IV] The background consists of a small number of galaxies that happen to be morphologically similar. The number of random pairs is approximately proportional to the area of an annulus, $d \Omega$, defined by a bin extending 
from $\Delta \theta$ to $\Delta \theta+\delta \theta$.

$$
d \Omega=\pi\left((\Delta \theta+\delta \theta)^{2}-\Delta \theta^{2}\right)
$$

which, for small bin sizes reduces to a very nearly linear rise with opening angle, $d \Omega=2 \pi \delta \theta \Delta \theta$.

\section{DATA SAMPLE:}

We analyze the GOODS Version 1.0 fits images taken with the ACS aboard HST [20]. Two fields are available, the Hubble Deep Field North (HDF-N) and the Chandra Deep Field South (CDF-S) which we refer to as the north and south fields respectively.

\section{A. Source identification}

SExtractor (Source Extractor) has, over the past several years, become the standard tool used in the analysis of space telescope data to identify sources. It has evolved from the "quick and dirty" solution envisioned by its author 21] to a program used worldwide by those conducting cosmological and astronomical research. We use the catalogs produced by an unmodified copy of SExtractor v2.5.0 in the analysis described here 22].

SExtractor's function is two-fold; namely, it processes FITS image files both to discover sources and, at the same time, to perform photometric calculations based on the data from those sources. Essentially, it locates stars, galaxies, and nebulae and makes estimates of their size, shape, brightness, and surrounding background exposure levels.

Calculating photometric quantities requires adjustment of a broad range of parameters specified in the SExtractor configuration file. For the GOODS I-band (F775W) north and south fields (the focus of this analysis), a specific set were chosen that maximize the efficiency of discovery while rejecting as many spurious objects as possible. A brief description of the relevant parameters and their values follows.

The ANALYSIS_THRESH and DETECT_THRESH parameters specify the level above background at which we set the pixel thresholds for the photometric/analytical and detection algorithms, respectively. For the analysis of the GOODS data, these two parameters are set to 1.0 times the RMS of the background level. The deblending threshold parameter, DEBLEND_NTHRESH, determines how aggressively SExtractor is in its attempts to subdivide an agglomeration of above-background pixels into subgroups corresponding to smaller, closely-spaced objects. The quantity DEBLEND_NTHRESH itself represents the number of brightness thresholds used in this procedure. We have used the (default) value of 32 . The associated parameter DEBLEND_MINCONT determines how bright a particular group of pixels must be to qualify as an independent object with the value 0 causing maximal deblending and the value 1 causing no deblending at all. For this analysis, we have set DEBLEND_MINCONT to 0.01. The minimum area a grouping of pixels must have in order to be considered an object is specified by the DETECT_MINAREA parameter. In this analysis this minimum area is 9 pixels.

The process of weighting in SExtractor is flexible, and many options exist. For our analysis we have found that the MAP_WEIGHT option, which requires a weight image accompanied by WEIGHT_THRESH of 0000000,0000000, gives the best results. Images, specified with WEIGHT_IMAGE on the SExtractor command line, are available alongside the scientific image files on the GOODS website. Apart from the parameter settings outlined here, all other inputs available in the SExtractor parameter file are set to their default values. These values are defined and discussed in detail in the SExtractor manual [21].

\section{B. Galaxy selection:}

The catalog created by SExtractor for the I-band fits images contains 51538 objects in the north field and 45 208 objects in the south field. To eliminate identification of spurious objects, we only select galaxies within a rectangular fiducial region where the exposure time is relatively uniform. The total area analyzed is $159.5 \mathrm{arcmin}^{2}$ in the north field and $155.4 \mathrm{arcmin}^{2}$ in the south field. We also remove stars from our own galaxy by requiring CLASS_STAR $<0.9$.

We further post-process our galaxy catalog by applying a procedure to identify the pixels in the image associated with each galaxy. The first step is to define a small but encompassing search region about each galaxy centroid and to smooth the region so that we are less sensitive to noise. We use a standard Lee filter in IDL for this (LEEFILT [23]). The second step is to find a bright pixel near the galaxy centroid. Then we look for neighboring pixels that are $1 \sigma$ above the noise threshold in the unsmoothed image and attach them to the centroid pixel cluster. By iteratively connecting neighbors that are above the noise threshold, we eventually get a cluster of pixels that we identify as the galaxy. This process sometimes merges neighboring galaxies. In the event that a cluster of pixels reaches the edge of the search region or that two galaxies merge, we raise the neighbor threshold to $2 \sigma$ and repeat the process until each galaxy is completely contained within the search region and does not contain the centroid from any other galaxy in the catalog. For a few very dim sources, the threshold is raised so high that there are no pixels left in the cluster and we remove these galaxies from the sample. After fiducial cuts, star removal, and pixel ID, the resulting catalogs contain 41358 galaxies in the north and 36328 galaxies in the south. 


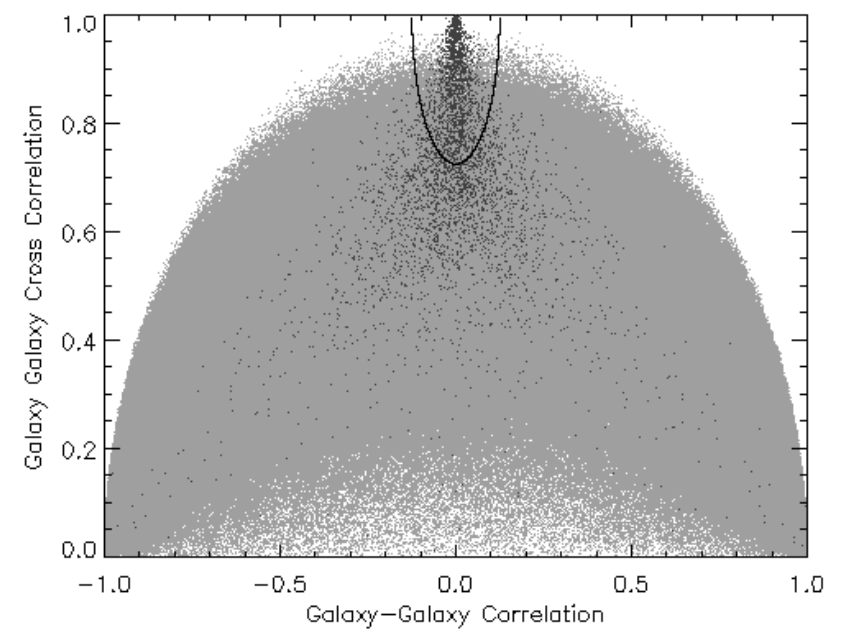

FIG. 3: Correlations for random pairs of galaxies in the GOODS data (light gray points) compared to simulated signal (black points). We define matched galaxy pairs as those within the elliptical line at the top of the plot where the correlation and cross-correlation are optimal.

\section{Galaxy-galaxy correlation:}

To calculate the morphological similarity between each pair of galaxies we rely on the correlation and crosscorrelation of the two galaxy images. This is a reasonably optimal way to assess the similarity of both brightness and shape. We first align the centroids and then calculate the correlation $(C O R R)$ and the cross-correlation $(X C O R R)$ of the pixels.

$$
\begin{gathered}
C O R R=\frac{\sum I_{1}\left(x_{i}, y_{i}\right)^{2}-\sum I_{2}\left(x_{i}, y_{i}\right)^{2}}{\sum I_{1}\left(x_{i}, y_{i}\right)^{2}+\sum I_{2}\left(x_{i}, y_{i}\right)^{2}} \\
X C O R R=\frac{2 \sum I_{1}\left(x_{i}, y_{i}\right) * I_{2}\left(x_{i}, y_{i}\right)}{\sum I_{1}\left(x_{i}, y_{i}\right)^{2}+\sum I_{2}\left(x_{i}, y_{i}\right)^{2}}
\end{gathered}
$$

where $I\left(x_{i}, y_{i}\right)$ is the intensity of each pixel in a galaxy and the subscript 1 or 2 refers to the galaxies being correlated. Perfectly correlated galaxies will have identical intensity distributions so that $C O R R=0$ and $X C O R R=1$. Measurement noise will smear the distributions out somewhat. Figure 3 shows the XCORR vs CORR distributions for both signal and background. The signal is concentrated as expected at the top and center but also has a long broad tail downward due to dim galaxies that are especially sensitive to noise. We have also found that near our detection threshold, galaxies tend to contain very few pixels and become round in appearance regardless of their true shape. The background is concentrated at $C O R R \sim \pm 1$ and $X C O R R \sim 0$, however, the statistical nature of the distribution extends between these limits in a semicircular pattern.

\section{Matched galaxy pair selection:}

We define matched galaxy pairs as those within the elliptical line drawn in Figure 3. This cut defines our definition of morphologically similar galaxies and was designed to maximize our signal pairs relative to background pairs. Although we would like to be more efficient for signals, we find that the signal outside this cut is swamped by background.

In this analysis, we consider pairs of galaxies with opening angles, $\Delta \theta<15^{\prime \prime}$. There are 3668 matched pairs in the north field and 2978 matched pairs in the south field with $\Delta \theta<15^{\prime \prime}$. This is compared to 5091 501 total pairings in the north field and 4180440 total pairings in the south field with $\Delta \theta<15^{\prime \prime}$. The correlation cuts therefore reduce the background by a factor of 1 400. A small region of the north field is shown in Fig. 2. The figure indicates that pairs passing our cuts appear fairly randomly distributed, consistent with random pairings that constitute our background.

\section{E. Pairs distribution:}

The binned distribution of matched galaxy pairs is shown in Fig. 4. The background shape is characterized by the distribution of all pairs of galaxies regardless of size and shape. Because strings with masses large enough to create opening angles greater than $7^{\prime \prime}$ have been ruled out [5, 9], we normalize the background distribution to the number of measured matched pairs between $7^{\prime \prime}$ and $15^{\prime \prime}$. This gives us a reliable estimate of the background at smaller opening angles. From the background, we observe that SExtractor merges galaxies with opening angles smaller than $0.4^{\prime \prime}$. At slightly larger opening angles there is a tiny excess of pairs created when SExtractor splits lumpy sources into two sources. We include this effect in the analysis, but note that the number of excess matched pairs is negligible. In our signal region, between $0.4^{\prime \prime}$ and $7^{\prime \prime}$, the $\chi^{2} /$ dof of the matched pairs to the background is 1.3 for the north field and 1.1 for the south field. Based on the scaled background distribution, we see no evidence for an excess of pairs at small opening angles.

\section{SIGNAL SIMULATION:}

The simulation of galaxy image pairs caused by the presence of a cosmic string is accomplished by laying down sample strings of a chosen energy-density/relativetilt $(\delta \sin \beta)$, and redshift $\left(z_{l}\right)$ across our fiducial region. We then statistically tally the resulting galaxies that would have been "lensed" if the string had existed. A galaxy is found to be lensed if the opening angle $(\Delta \theta)$ calculated in Eq. 2 is sufficient as to place the imagegalaxy on the side of the string opposite the true-galaxy. The accumulated samples result in a catalog of lensed 

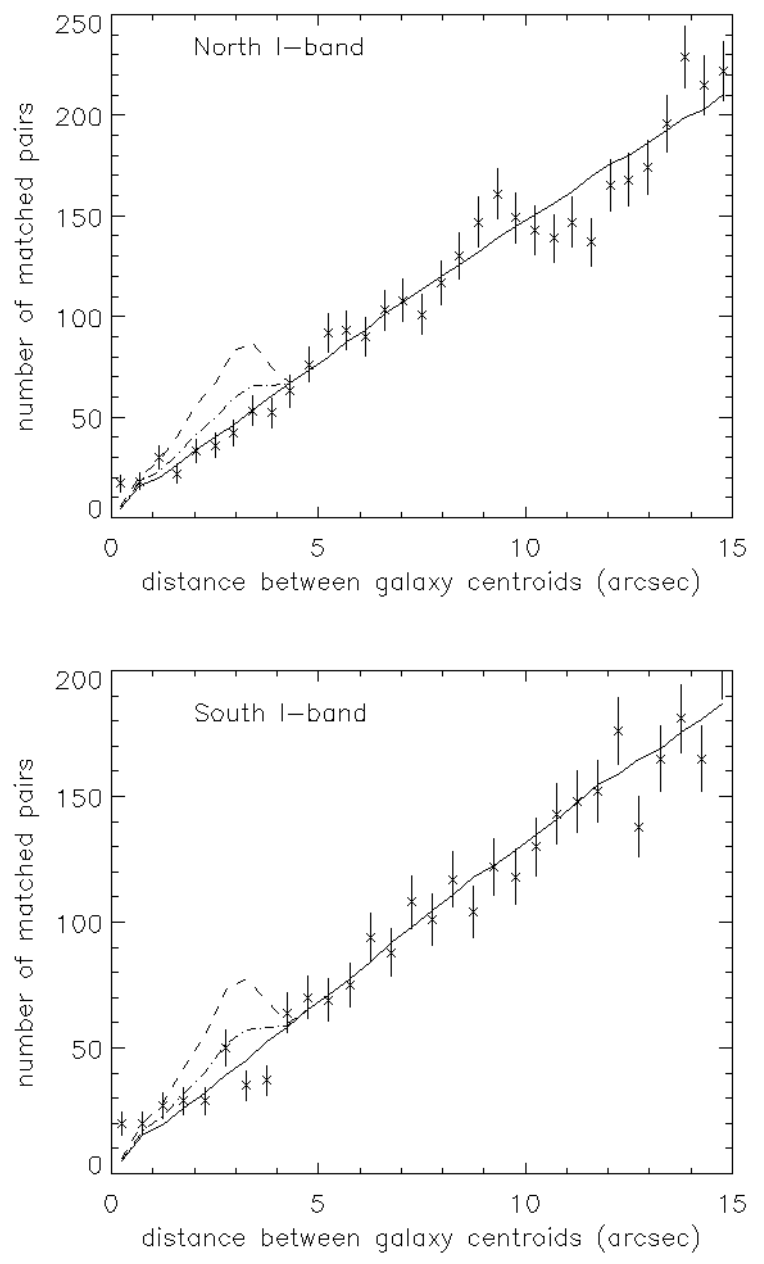

FIG. 4: Pairs of galaxies in the GOODS north- and southfield data (points) compared to background (solid line) and one example of a simulated string (dashes). The upper simulated string is the total number of pairs expected from the simulation with string redshift of 1.2 and $\delta \sin \beta$ of $6^{\prime \prime}$. The lower simulated string includes measurement inefficiencies.

galaxies which also includes other pertinent information about each lensing event, such as the opening angle. This information is then used for computing signal densities as shown in Fig. 5. To reduce numerical uncertainty, we simulate as many independent string trials as are necessary until the accumulated total of lensed sources $\left(N_{s r c}\right)$ is 10,000 . That is, $1 / \sqrt{N_{s r c}}<0.01$ [16, 24].

The ratio $\mathrm{D}_{l s} / \mathrm{D}_{o s}$ is a critical factor for determining opening angle, and hence, whether or not a lensed image results. We simulate these distance factors for a $\Lambda \mathrm{CDM}$ cosmology [19] with $H_{0}=73 \mathrm{~km} /(\mathrm{s} \mathrm{Mpc}), \Omega_{\Lambda}=0.76$ and $\Omega_{M}=0.24$. The ratio of distance factors is illustrated in Fig. 6. Knowledge of source redshift is essential in its calculation, however, redshift data for the GOODS survey is not presently available. This problem is resolved by assigning redshifts to each source based on their SExtractor I-band magnitude (MAG_AUTO) as outlined in

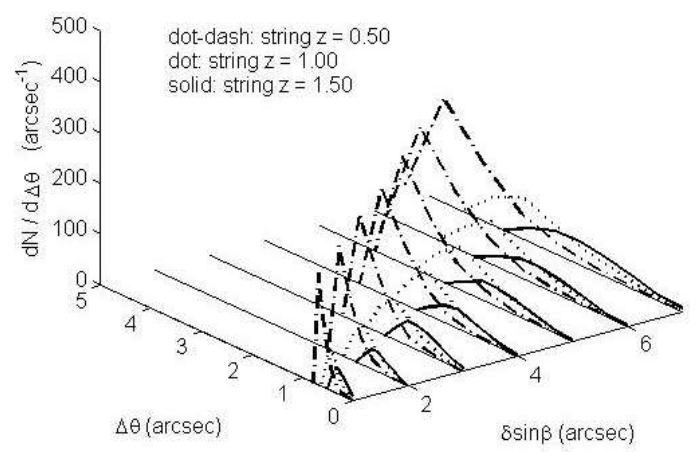

FIG. 5: Various examples of simulated signals where $d N / d \Delta \theta$ represents the number of lensed galaxy pairs per angular separation distance as a function of cosmic string parametersenergy-density $(\delta \sin \beta)$ and redshift $\left(z_{l}\right)$. In these simulations, the number of signal events are scaled to a cosmic string with a total length of 10 arcminutes.

[25]. The source-number density is found to be reasonably approximated by:

$$
\frac{d N_{s r c}\left(z_{s}\right)}{d z} \propto z^{2} e^{-\left(z / z_{m}\right)^{2}}
$$

where

$$
z_{m}=0.722+0.149(I-22.0)
$$

Figure 7 is the result of applying Eq. 6 to the GOODS north field catalog. Each source in the catalog is assigned a redshift that is distributed according to the model. The figure shows the number density of galaxies as a function of redshift in the GOODS north field for integer bins of I-band magnitude.

\section{DETECTION EFFICIENCIES:}

There are a number of reasons why some lensed pairs would not be detected. Figure 8 shows a summary of the efficiencies as a function of the opening angle between the galaxies and the string redshift.

\section{A. Survey edges}

Survey edges will occasionally be a factor when either the direct or lensed image of the galaxy does not land inside our fiducial region. We fit the shape of the background distibution in Fig. 4 with a second order polynomial. If the survey were infinitely large, the distribution would rise perfectly linearly as described in the overview,

$$
d N / d \Delta \theta \propto 2 \pi \delta \theta \Delta \theta
$$




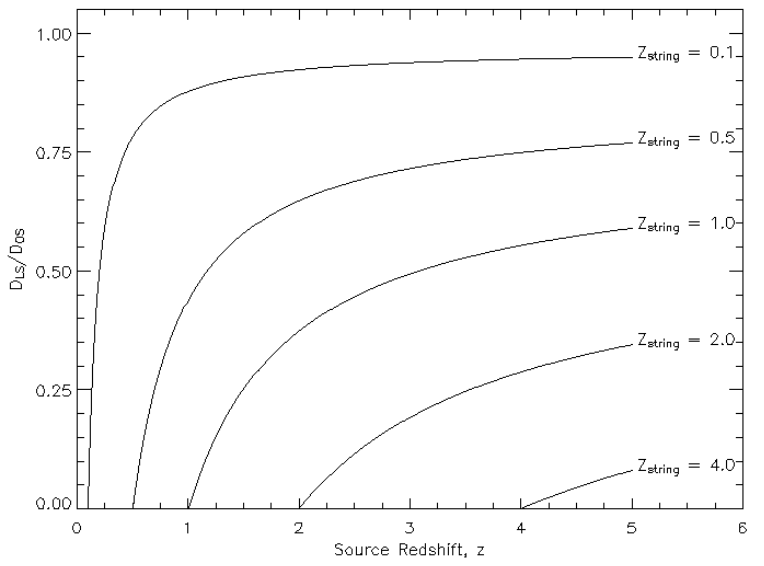

FIG. 6: The ratio $\mathrm{D}_{l s} / \mathrm{D}_{o s}$ is a multiplicative factor which affects the opening angle in Eq. 2, Here we plot it for a flat $\Lambda \mathrm{CDM}$ cosmology, $H_{0}=73 \mathrm{~km} /(\mathrm{s} \mathrm{Mpc}), \Omega_{\Lambda}=0.76, \Omega_{M}=$ 0.24 .

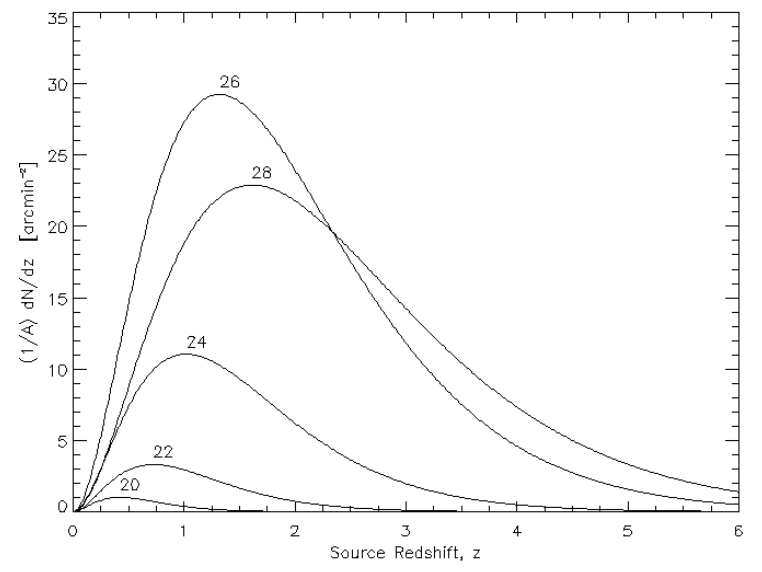

FIG. 7: Modeled GOODS north field source redshift probability distribution function (PDF) as a function of redshift and I-band magnitude (MAG_AUTO - shown on each curve). Distributions are scaled to the north field area $(\mathrm{A} \simeq 160$ $\left.\operatorname{arcmin}^{2}\right)$.

where $\delta \theta$ is the bin width and $\Delta \theta$ is the opening angle between the galaxies. Because the measured distribution falls slightly below the line at the largest opening angles, we estimate the inefficiency due to the edges of our survey as the ratio of the quadratic and linear terms in the $2^{\text {nd }}$ order fit to the background. As shown in Fig. 8, the inefficiency due to edges is never more than $5 \%$ in our signal region.

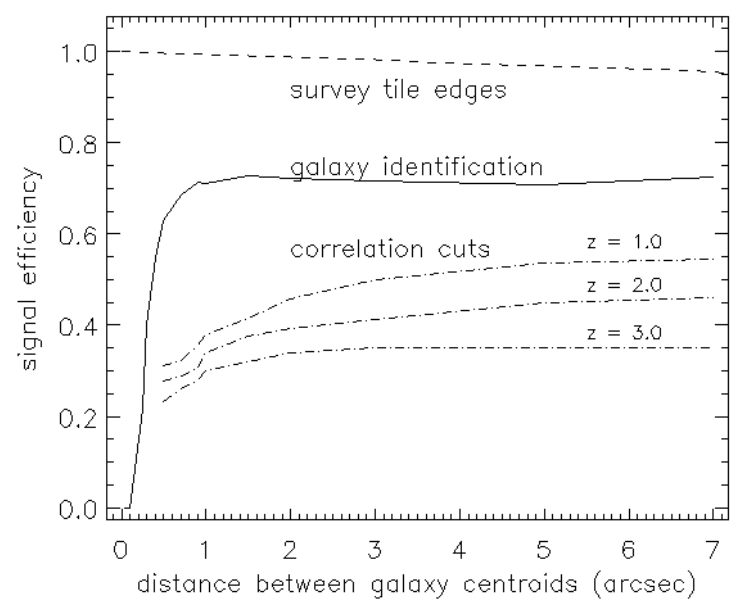

FIG. 8: Efficiency of detecting pairs of galaxies lensed by a cosmic string as a function of pair opening angle. The correlation cut efficiencies are dependent on redshift as indicated. The other efficiencies are only weakly dependent on redshift.

\section{B. Galaxy pair detection and merging}

Given one galaxy, we estimate the probability that its lensed partner is found by embedding galaxies back into the original fits images. We use simulated strings to determine which galaxies are lensed in the image. So that the embedded galaxy does not contribute excess noise to the image, we smooth it with the Lee Filter [23] in IDL prior to adding its intensity to the image intensity. The smoothing makes the galaxy slightly dimmer than its original, and we note that this technique slightly underestimates the efficiency at large opening angles which results in our limits being slightly conservative. We then pass the image, including the embedded galaxies through the entire analysis chain.

The efficiencies are calculated by comparing the number of galaxies identified by the analysis chain to the number identified before embedding plus the number embedded. We attribute the inefficiency at large opening angles to dim galaxies that are lost due to measurement noise. At small opening angles SExtractor merges the galaxies into a single object. The galaxy identification efficiency is only weakly dependent on the redshift of the string.

\section{Correlation cuts}

In addition to the inefficiencies inherent in finding the lensed galaxy, there are inefficiencies due to the correlation estimators. Noise can bias the CORR and $X C O R R$ variables. Using embedded galaxies that have been passed through the analysis chain, we compare the number that pass the correlation cuts to the number of embedded galaxies that were detected. The efficien- 
cies drop for small opening angles due to galaxies near the edge of our lensing corridor which are only partially lensed. The correlation efficiency also shows a relatively strong dependence on the redshift of the string. We attribute this to the fraction of dim galaxies lensed in the sample. A high redshift string has a larger fraction of dim galaxies behind it than does a low redshift string.

\section{RESULTS:}

The distribution of matched galaxy pairs was shown in Fig. 4, It rises nearly linearly as expected from Eq. 8 with a slight inefficiency due to the edges of the survey shown in Fig. 8, For comparison, pairs from a cosmic string at a redshift of 1.2 and $\delta \sin \beta$ of $6^{\prime \prime}$ are included on the plot normalized to the mean string length of $13.1^{\prime}$ in the north field and $11.7^{\prime}$ in the south field. The upper curve is the simulated signal without detection inefficiencies scaled from Fig. 5. The lower curve includes the measurement inefficiencies from Fig. 8.

We compare a wide variety of predicted cosmic string signals to the data to determine limits. For each signal, we integrate the signal to find $n_{s}$ pairs. We then integrate the matched pairs and the background curve over the opening angles from $0.4^{\prime \prime}$ where the efficiency estimates are reliable up to the point where there are no more signal pairs to determine $n_{o b s}$ and $n_{b}$. We report classical single-sided Neyman confidence limits, (C.L.), where the probability of finding a signal is limited to a region:

$$
P\left(n_{o b s}<n_{b}+n_{l i m} \mid \sigma\right)=\text { C.L. }
$$

The estimator of the experimental fluctuations is $\sigma$, the mean background is $n_{b}$, and the minimum number of signal events that would be consistent with background fluctuations is $n_{\text {lim }}$. That is, $n_{s}>n_{\text {lim }}$ is excluded and $n_{s}<n_{\text {lim }}$ is not. Since our backgrounds are relatively large, we express the probability as a Gaussian distribution with $\mu=\left(n_{o b s}-n_{b}\right)$ and $\sigma=\sqrt{n_{o b s}}$.

$$
P\left(n_{x} \mid \mu, \sigma\right)=\frac{1}{\sqrt{2 \pi} \sigma} \exp \left(-\left(\mu-n_{x}\right)^{2} / 2 \sigma^{2}\right)
$$

This is a Gaussian with mean near zero and a width that represents the statistical fluctuations in the data. The limit, $n_{\text {lim }}$, is then the value of $n_{x}$ for which $95 \%$ of the area under the Gaussian is left of $n_{x}$. This is $P\left(n_{x}<n_{\text {lim }} \mid \mu, \sigma\right)=$ C.L.. The resulting $95 \%$ confidence limits are shown in Fig. 9, The limits extend from $0.5^{\prime \prime}<\delta \sin \beta<7^{\prime \prime}$. Taking the mean tilt of a string with respect to the observer to be $\langle\sin \beta\rangle=2 / \pi$ we relate the opening angle to the mass scale by $G \mu / c^{2}=\delta<$ $\sin \beta>/(8 \pi)$ shown on the right-hand axis. We see no evidence for cosmic strings out to a redshift greater than 0.5 in the north field and greater than 0.3 in the south field and place a limit on $G \mu / c^{2}<3.0 \times 10^{-7}$ at $95 \%$ C.L. The north field limits extend to higher redshifts than the

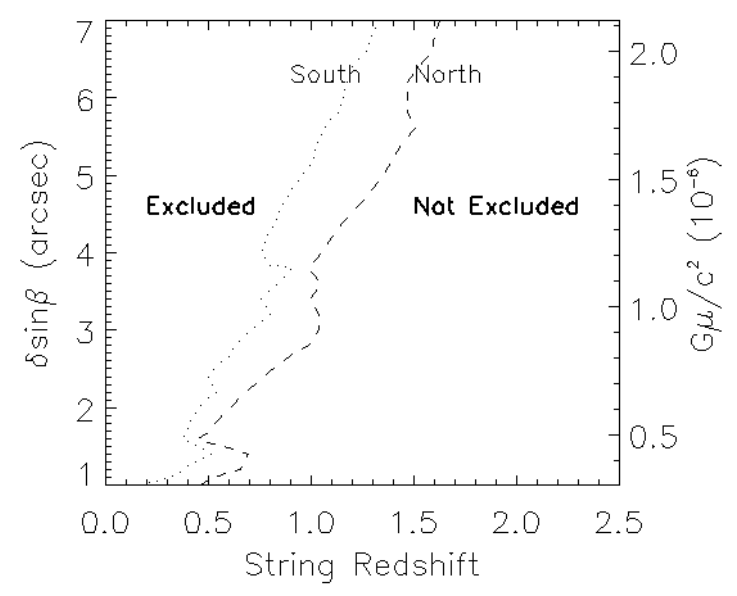

FIG. 9: Confidence limits at 95\% for lensed galaxies produced by a cosmic string as a function of the string's mass and redshift. The north field limits extend to higher redshifts due to statistical fluctuations of signal and background, and also because the mean string length across the survey is a bit longer and the number density of galaxies is somewhat higher.

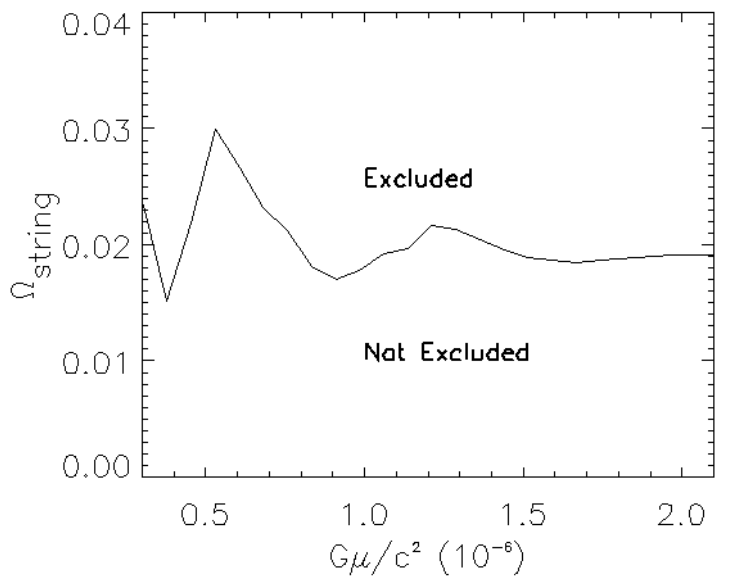

FIG. 10: Confidence limits at $95 \%$ on $\Omega_{\text {String }}$ as a function of the string mass.

south field limits due to statistical fluctuations of signal and background and also because the north field has a longer mean string length across the survey and a higher number density of galaxies.

It is highly unlikely that a string passes through both the north and south fields and we therefore treat the two surveys as uncorrelated measures of the string density. If strings are rare occurrences, it is possible that none would appear in the GOODS fields and that other survey fields may yield different results. We can interpret our result, however, as excluding the possibility that 3 strings would be located in any one field of view with $95 \%$ confidence. 
This corresponds as a string density

$$
\Omega_{\text {strings }}=\frac{\rho_{\text {strings }}}{\rho_{\text {critical }}} \simeq \frac{N \mu}{\text { fov } \eta^{2} / 3} \times \frac{8 \pi G}{3 H_{0}^{2}}
$$

where $N=3$ strings, $\mu$ is the mass per length of the string, fov is the survey field of view $\left(159.5 \operatorname{arcmin}^{2}\right.$ in the north and $155.4 \mathrm{arcmin}^{2}$ in the south), and $\eta$ is the comoving distance. We combine the north and south fields by averaging the string length within the field of view and summing the volume of the two fields. Figure 10 shows the string densities excluded by this method. The limit excludes a string density that is $2 \%$ of the critical density. The variation observed is due to statistical fluctuations in background and is sensitive to the smallangle signal efficiencies.

\section{CONCLUSION:}

We use the GOODS HDF-N and CDF-S fields to search for cosmic strings. We find no evidence for the existence of the gravitational lensing signature. We have included the observational efficiencies in our analysis using a new technique of embedding galaxies based on a robust string simulation. Our results are summarized in Figs. 9 and 10. From this search we conclude with $95 \%$ confidence that $G \mu / c^{2}<3.0 \times 10^{-7}$ out to redshifts greater than 0.5 and that $\Omega_{\text {strings }}<0.02$. We note that these results are for long straight strings, but also exclude moderately curved strings as long as there are no kinks in the field of view.
These are the first reported limits using the gravitational lensing signature and are consistent with recently reported sensitivities of such searches [17]. Our excluded masses are smaller than those excluded by direct CMB searches [9, 10]. Our limits on $\Omega_{\text {string }}$ are still weaker, however, due to the full-sky coverage and high redshift of the CMB. Our excluded masses are larger than those reported by parameter fits to the CMB [7] as well as gravitational wave searches [11, 12], but these depend on features of the modeling which do not affect our direct search. We note that recently rekindled interest in microlensing signatures [26, 27] is motivated by the challenge of detecting smaller mass cosmic strings over a larger fraction of the sky out to high redshift. We are now in the infancy of wide-field high resolution surveys and are excited about the prospect of pursuing these techniques with larger surveys such as COSMOS 28] and SNAP[29].

\section{ACKNOWLEDGEMENTS:}

We would like to thank Lluvia Zuniga, Kirsten Howley, Wes Salpiea, and Mia Ihm for their early involvement in this project. E. Albin was partially supported by the SULI summer program at LBNL. K. James was partially supported by the Cal Poly SLO Department of Physics. J. L. Christiansen and E. Albin were partially supported by the Cal Poly SLO Provost's Extramural Funding Initiative.
[1] T. Kibble, J. Phys. A 9, 1387 (1976).

[2] J. Polchinski, hep-th/0412244.

[3] M. Hindmarsh and T. Kibble, Rep. Prog. Phys. 58, 477 (1995).

[4] M. Sakellariadou, hep-th/0602276.

[5] L. Pogosian, S. Tye, I. Wasserman, and M. Wyman, Phys. Rev. D 68, 023506 (2003).

[6] L. Pogosian, M. Wyman, and I. Wasserman, J. Cosmol. Astropart. 09, 008 (2004).

[7] M. Wyman, L. Pogosian, and I. Wasserman, Phys. Rev. D 73, 089905 (2006).

[8] N. Bevis, M. Hindmarsh, M. Kunz, and J. Urrestilla, Phys. Rev. D 75, 065015 (2007).

[9] E. Jeong and G. Smoot, Astrophys. J. 624, 21 (2005).

[10] A. Lo and E. Wright, astro-ph/0503120.

[11] F. Jenet et al., Astrophys. J. 653, 1571 (2006).

[12] X. Siemens, V. Mandic, and J. Creighton, Phys. Rev. Lett. 98, 111101 (2007).

[13] M. Sazhin et al., Mon. Not. Roy. Astron. Soc. 343, 353 (2003).

[14] M. Sazhin et al., astro-ph/0406516.

[15] E. Agol, C. Hogan, and R. Plotkin, Phys. Rev. D 73, $087302(2006)$.
[16] M. Oguri and K. Takahashi, Phys. Rev. D 72, 085013 (2005).

[17] M. Gasparini et al., astro-ph/0710.5544.

[18] P. Peebles, Principles of Physical Cosmology (Princeton University Press, 1993).

[19] B. Ryden, Introduction to Cosmology (Pearson Education Inc., 2003).

[20] M. Giavalisco and others (GOODS Team), Astrophys. J. L93, 600 (2004).

[21] E. Bertin, Sextractor v2.4 user's manual (2005).

[22] E. Bertin and Arnouts, Astron. and Astron. Suppl. Ser. 117, 393 (1996).

[23] J. Lee, Opt. Eng. 25, 636 (1986).

[24] D. Huterer and T. Vachaspati, Phys. Rev. D 68, 041301 (2003).

[25] R. Massey et al., Astron.J. 127, 3089 (2004).

[26] K. Mack, H. Wesley, and L. King, Phys. Ref. D 76, 123515 (2007).

[27] K. Kuijken, X. Siemens, and T. Vachaspati, astro$\mathrm{ph} / 0707.2971$.

[28] N. Scoville et al., astro-ph/0612305.

[29] G. Aldering et al., astro-ph/0405232. 